\title{
Paeoniflorin protects PC12 cells from oxygen-glucose deprivation/reoxygenation-induced injury via activating JAK2/STAT3 signaling
}

\author{
ZHUO ZHANG and WEIMIN YANG
}

Department of Neurology, The First Affiliated Hospital of Zhengzhou University, Zhengzhou, Henan 450000, P.R. China

Received December 2, 2020; Accepted February 26, 2021

DOI: $10.3892 /$ etm.2021.10004

\begin{abstract}
Ischemic stroke is the most common type of stroke, and it has become a major health issue as it is characterized by high mortality and morbidity rates. Paeoniflorin $(\mathrm{PF})$ is a natural compound and the main active ingredient of Radix Paeoniae. The aim of the present study was to investigate the role of PF in oxygen-glucose deprivation/reoxygenation (OGD/R)-induced injury of PC12 cells and its association with the Janus kinase 2 (JAK2)/signal transducer and activator of transcription 3 (STAT3) pathway. An in vitro model of OGD/R injury was established in PC12 cells. Subsequently, Cell Counting Kit- 8 assay and ELISA were used to evaluate cell viability and the secretion of inflammatory factors, respectively, in PC12 cells subjected to OGD/R and treated with PF. The levels of oxidative stress indicators and inflammatory factors were measured using corresponding commercial kits. In addition, the apoptosis rate of PC12 cells subjected to OGD/R and treated with PF was determined by flow cytometry, and the expression of apoptosis-related proteins was analyzed by western blotting. Additionally, the expression levels of JAK2/STAT3 pathway-related proteins were also evaluated. The cell viability, levels of oxidative stress, inflammation and apoptosis were also measured in OGD/R-induced PC12 cell injury models following co-treatment of cells with PF and FLLL32, a specific inhibitor of JAK2/STAT3 signaling. Cell viability was reduced, while oxidative stress and inflammation were increased after OGD/R-induced injury. However, the treatment of cells with PF significantly enhanced cell viability, and alleviated oxidative stress, inflammation and apoptosis of OGD/R-treated PC12 cells. Furthermore, PF activated the JAK2/STAT3 signaling pathway. Following FLLL32 intervention, the effects of PF on oxidative stress, inflammation and
\end{abstract}

Correspondence to: Dr Weimin Yang, Department of Neurology, The First Affiliated Hospital of Zhengzhou University, 1 Jianshe East Road, Zhengzhou, Henan 450000, P.R. China

E-mail: yangweimywm@163.com

Key words: ischemic stroke, paeoniflorin, inflammation, apoptosis, oxygen-glucose deprivation/reoxygenation apoptosis of OGD/R-treated PC12 cells were reversed. In conclusion, the findings of the present study suggested that $\mathrm{PF}$ may protect PC12 cells from OGD/R-induced injury via activating the JAK2/STAT3 signaling pathway, thus providing novel insight into the mechanism through which PF may alleviate ischemic stroke and indicating a potential strategy for ischemic stroke treatment.

\section{Introduction}

Ischemic stroke is a major type of stroke that is characterized by high mortality and morbidity rates (1). The oxygen and glucose deprivation (OGD) that results from complete or partial blockade of arterial blood supply to the brain is considered as the leading cause for the occurrence of ischemic stroke (2). Ischemia-reperfusion (I/R) injury, which is defined as the restoration of blood supply after a given duration of ischemia, is a common characteristic of ischemic stroke (3). Although the incidence of ischemic stroke is high, effective treatment strategies for this disease are still lacking.

Paeoniflorin (PF; Fig. 1A), a natural compound, is the main active ingredient of Radix Paeoniae (4). A previous study demonstrated that PF protected HT-22 cells from $\mathrm{H}_{2} \mathrm{O}_{2}$-induced oxidative injury via regulating the expression of microRNA(miR)-135a(5).Inaddition,6'-O-galloylpaeoniflorin, the galloylated derivative of PF isolated from peony root, attenuated neuroinflammation and oxidative stress in a cerebral I/R injury rat model via activating the PI3K/AKT/nuclear factor erythroid-2-like 2 signaling pathway (6). Compelling evidence has indicated that PF combined with $\beta$-ecdysterone protected PC12 cells against neurotoxicity triggered by rotenone (7). Additionally, 6-hydroxydopamine-induced PC12 cell apoptosis was suppressed by PF via blocking the reactive oxygen species-induced protein kinase $\mathrm{C} \delta / \mathrm{NF}-\kappa \mathrm{B}$ signaling pathway (8). Another study revealed that the function of PF in preventing mitochondrial dysfunction may alleviate cytotoxicity in glutamate-induced PC12 cells (9). Importantly, it has also been reported that the natural neuroprotector PF can inhibit a number of pro- and anti-inflammatory signals in differentiated PC12 cells (10).

It has been suggested that Janus kinase 2 (JAK2), a crucial factor involved signaling through a variety of cytokine receptors, phosphorylates signal transducer and activator of transcription 3 (STAT3) upon activation (11). A 
previous study indicated a strong association between the phosphorylation/activation of the JAK2/STAT3 pathway and a neuroprotection-related signaling pathway (12). Blocking the JAK2/STAT3 signaling pathway by PF may protect the kidneys ofdiabeticrats(13). Furthermore, the melatonin-mediated regulation of the miR-26a-5p/neuron-restrictive silencer factor and JAK2/STAT3 pathways may attenuate cerebral $\mathrm{I} / \mathrm{R}$ injury via alleviating inflammation and oxidative stress (14). In addition, the activation of the JAK2/STAT3 pathway by Src homology 2B adaptor protein 1 protected PC12 cells from oxygen-glucose deprivation/reoxygenation (OGD/R)-induced apoptosis (15). Therefore, it was hypothesized that $\mathrm{PF}$ may play a significant role against OGD/R-induced PC12 cell injury via regulating the JAK2/STAT3 pathway.

The aim of the present study was to investigate the function of PF in OGD/R-induced PC12 cell injury and the role of the JAK2/STAT3 signaling pathway in this process, in order to elucidate whether PF may be considered as a promising candidate for the treatment of ischemic stroke.

\section{Materials and methods}

PC12 cell culture and treatment. The rat pheochromocytoma cell line, PC12, was obtained from the American Type Cell Culture Collection. The cells were cultured in DMEM (Gibco; Thermo Fisher Scientific, Inc.) supplemented with 10\% FBS (Gibco; Thermo Fisher Scientific, Inc.), $100 \mathrm{U} / \mathrm{ml}$ penicillin and streptomycin at $37^{\circ} \mathrm{C}$ in a humidified incubator containing $5 \% \mathrm{CO}_{2}$. PF (purity, $>98 \%$ ) was purchased from the National Institute for the Control of Pharmaceutical and Biological Products (Beijing, China). PF was dissolved in DMSO (Sigma-Aldrich; Merck KGaA) to a final concentration of $200 \mu \mathrm{M}$. A total of $100 \mu \mathrm{l} \mathrm{PF}(200 \mu \mathrm{M})$ was diluted to 100 , 50 and $25 \mu \mathrm{M}$ PF by adding into 100,300 and $700 \mu \mathrm{l}$ DMEM, respectively. The final concentration of DMSO in the cultures was $<1 \%$. The cells were detached and re-seeded into six-well plates $\left(1 \times 10^{6}\right.$ cells per well) for the subsequent experiments. Finally, the cells were left untreated or were pre-treated with various concentrations of PF for $24 \mathrm{~h}$, followed by treatment with FLLL32, a specific inhibitor of JAK2/STAT3 signaling, at a dose of $25 \mu \mathrm{M}$ for an additional $24 \mathrm{~h}$.

Establishment of OGD/R injury model. PC12 cells were cultured in Earle's Balanced Salt Solution (Sigma-Aldrich; Merck KGaA) without glucose to induce cell ischemia. The cells were maintained in a three-gas incubator containing $94 \% \mathrm{~N}_{2}, 5 \% \mathrm{CO}_{2}$ and $1 \% \mathrm{O}_{2}$ at $37^{\circ} \mathrm{C}$ for $4 \mathrm{~h}$. Subsequently, the medium was discarded and replaced with normal medium supplemented with $10 \%$ FBS, and cells were reoxygenated in a normal atmosphere for an additional $24 \mathrm{~h}$ for the establishment of the reperfusion model.

Cell Counting Kit-8 (CCK-8) assay. To determine PC12 cell viability, the cells were seeded into a 96 -well plate at a density of $3 \times 10^{4}$ cells per $100 \mu \mathrm{l}$ medium in each well. Following incubation for $24 \mathrm{~h}, 10 \mu \mathrm{l} \mathrm{CCK}-8$ reagent was added into each well, and the plate was incubated at $37^{\circ} \mathrm{C}$ for an additional $4 \mathrm{~h}$. The absorbance of each well was measured at $450 \mathrm{~nm}$ using a microplate reader (BioTek Instruments, Inc.).
Determination of oxidative stress. Briefly, $2 \times 10^{5} \mathrm{PC} 12$ cells/well were seeded into 96-well plates. The activity of lactate dehydrogenase (LDH; cat. no. A020-2-2), myeloperoxidase (MPO; cat. no. A044-1-1) and superoxide dismutase (SOD; cat. no. A001-3-2) was determined using the corresponding kits (Nanjing Jiancheng Bioengineering Institute), according to the manufacturers' instructions. Subsequently, the absorbance was determined at $450 \mathrm{~nm}$ (LDH and SOD) or $460 \mathrm{~nm}$ (MPO) using a microplate reader (Bio-Rad Laboratories, Inc.).

Determination of the levels of inflammatory factors. ELISA kits were used to measure the levels of TNF- $\alpha$ (cat. no. F16960), IL-6 (cat. no. F15870) and IL-10 (cat. no. F15900) in the cell culture medium. The cells were first treated with $500 \mu \mathrm{l}$ ice-cold carbonate buffer (100 mM Na $\left.\mathrm{CO}_{3}, 50 \mathrm{mM} \mathrm{NaCl}, \mathrm{pH} 11.5\right)$ supplemented with protease inhibitors, and were then dissociated using an ultrasonic cell disruption system (Ningbo Haishu Kesheng Ultrasonic Equipment Co., Ltd.). The mixture was centrifuged at $12,000 \mathrm{x}$ g for $45 \mathrm{~min}$ at $4^{\circ} \mathrm{C}$. The supernatant was then collected, and the levels of the inflammatory factors were measured according to the manufacturer's instructions (Shanghai Xitang Biotechnology Co., Ltd.).

Flow cytometric analysis. Flow cytometry using the Annexin V-FITC apoptosis kit (Beyotime Institute of Biotechnology) was performed to determine cell apoptosis. Following a series of treatments as aforementioned, PC12 cells were collected by centrifugation $(200 \mathrm{x} \mathrm{g} ; 10 \mathrm{~min}$; room temperature), then washed twice by ice-cold PBS, resuspended in $195 \mu \mathrm{l}$ pre-chilled $1 \mathrm{X}$ Annexin V binding buffer. Subsequently, cells were doubled-stained with $5 \mu 1$ Annexin V-FITC and $5 \mu \mathrm{l}$ propidium iodide (PI) for $15 \mathrm{~min}$ in the dark at room temperature according to the manufacturer's instructions. Subsequently, cell apoptosis of each sample was assessed by flow cytometer (BD Accuri ${ }^{\mathrm{TM}}$ C6; BD Biosciences). The data were analyzed using FlowJo software (version 7.6.1; FlowJo LLC). Annexin V and PI single-stained positive cells were used to regulate compensation (as control). The sum of apoptosis rate in right upper quadrant $(\mathrm{Q} 2$, late apoptotic cells) and right lower quadrant (Q3, early apoptotic cells) were considered as the cell apoptosis rate. The apoptotic cells were expressed as a percentage of the total number of cells.

Western blot analysis. Total proteins were extracted from PC12 cells using RIPA lysis buffer (Beyotime Institute of Biotechnology) and the protein concentration was determined by a BCA Protein Assay kit (Beyotime Institute of Biotechnology). The protein samples (40 $\mu \mathrm{g} / \mathrm{lane}$ ) were then separated by $10 \%$ SDS-PAGE and transferred onto a PVDF membrane (EMD Millipore). Subsequently, the membrane was blocked with $5 \%$ skimmed milk for $2 \mathrm{~h}$ at room temperature, washed with Tris-buffered saline containing 0.2\% Tween-20 (TBST; Boster Biological Technology), and incubated with the primary antibodies at $4^{\circ} \mathrm{C}$ overnight. After washing with TBST, the membrane was incubated with a goat anti-rabbit horseradish peroxidase (HRP)-conjugated secondary antibody (1:3,000; cat. no. 7074S; Cell Signaling Technology, Inc.) or horse anti-mouse HRP-conjugated secondary antibody (1:3,000; cat. no. 7076S; Cell Signaling Technology, Inc.) at room temperature for $2 \mathrm{~h}$. Finally, protein 

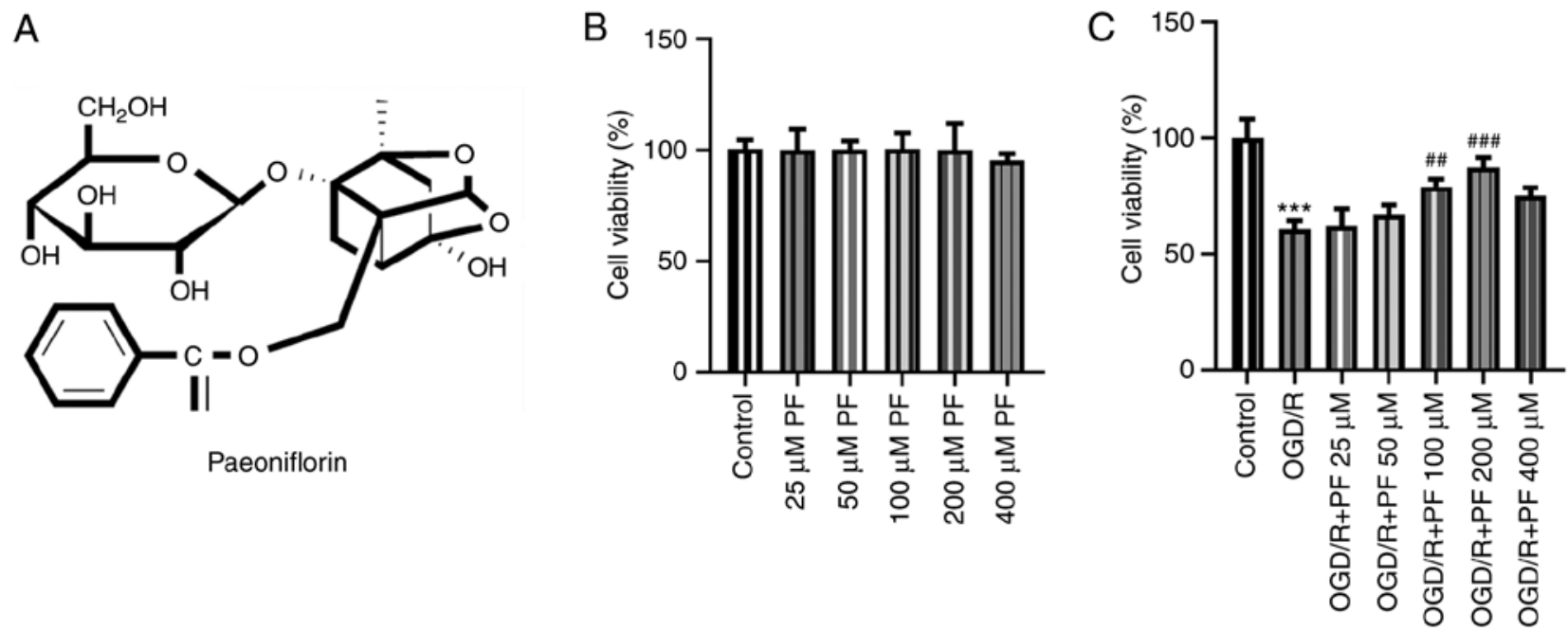

Figure 1. PF enhances the viability of OGD/R-treated PC12 cells. (A) Chemical structure of PF. (B) CCK-8 assay was used to determine the viability of PC12 cells treated with increasing doses of PF. (C) Following establishment of OGD/R-induced PC12 cell injury model, the apoptosis rate of cells treated with increasing doses of PF was determined using CCK-8 assay. ${ }^{* * * *} \mathrm{P}<0.001$ vs. the control group; ${ }^{\# \#} \mathrm{P}<0.01,{ }^{\# \# \#} \mathrm{P}<0.001$ vs. the OGD/R group. $\mathrm{PF}$, paeoniflorin; OGD/R, oxygen-glucose deprivation/reoxygenation; CCK-8, Cell Counting Kit-8.

bands were visualized using an enhanced chemiluminescence substrate (Pierce; Thermo Fisher Scientific, Inc.) on a chemiluminescence imaging equipment (Ultra-Lum, Inc.). The proteins bands were quantified using the ImageJ software (version 1.52r; National Institutes of Health). The gray value of the target protein was normalized to that of GAPDH. The following primary antibodies were used: Anti-Bax (cat. no. 14796S; 1:1,000), anti-cleaved caspase-9 (cat. no. 20750S; 1:1,000), anti-cleaved poly(adenosine diphosphate-ribose) polymerase (PARP) (cat. no. 9185S; 1:1,000), anti-p-JAK2 (cat. no. 3776S; 1:1,000), anti-JAK2 (cat. no. 3230T; 1:1,000), anti-p-STAT3 (cat. no. 9145S; 1:1,000), anti-STAT3 (cat. no. 4904T; 1:1,000) and anti-GAPDH (cat. no. 5174S; 1:1,000). All antibodies were obtained from Cell Signaling Technology, Inc., apart from anti-Bcl-2 (cat. no. sc-7382; 1:1,000), which was purchased from Santa Cruz Biotechnology, Inc.

Statistical analysis. Statistical analysis was performed using GraphPad Prism (version 6.0; GraphPad Software, Inc.) and data are expressed as the mean $\pm \mathrm{SD}$. All experiments were performed three times. Statistical comparisons among multiple groups were analyzed using one-way ANOVA followed by a Tukey's post hoc test. $\mathrm{P}<0.05$ was considered to indicate a statistically significant difference.

\section{Results}

$P F$ enhances the viability of PC12 cells subjected to $O G D / R$. The viability of $\mathrm{PC} 12$ cells was determined to evaluate the effect of different concentrations of PF. As shown in Fig. 1B, treatment of PC12 cells with $25,50,100$ or $200 \mu \mathrm{M}$ PF did not affect cell viability, whereas treatment with $400 \mu \mathrm{M}$ PF decreased PC12 cell viability. After the establishment of the OGD/R cell model, the viability of PC12 cells was notably decreased compared with the control group, and it was gradually restored following treatment with increasing concentrations of $\mathrm{PF}(25,50,100$ or $200 \mu \mathrm{M})$. This finding suggested that PF could increase the viability of OGD/R-treated PC12 cells (Fig. 1C). Since treatment with $400 \mu \mathrm{M}$ PF promoted $\mathrm{PC} 12$ cell injury and exerted a weaker effect on OGD/R-treated PC1 2 cells compared with $200 \mu \mathrm{M} \mathrm{PF}$, doses of 25-200 $\mu \mathrm{M} \mathrm{PF}$ were selected for the subsequent experiments.

PF alleviates oxidative stress, inflammation and apoptosis in OGD/R-treated PC12 cells. To determine whether PF could mitigate the OGD/R-induced cell injury, the levels of oxidative stress indicators and inflammatory factors were determined. The levels of LDH and MPO were significantly increased, while those of SOD were markedly reduced by $\mathrm{OGD} / \mathrm{R}$. Treatment with increasing concentrations of $\mathrm{PF}$ reduced the levels of LDH and MPO, and increased those of SOD (Fig. 2A-C). Additionally, the ELISA results demonstrated that the levels of TNF- $\alpha$ and IL- 6 were increased and those of IL-10 were decreased in the OGD/R group compared with the control group, while treatment with PF had the opposite effect (Fig. 2D-F). Overall, the aforementioned results suggested that PF could attenuate oxidative stress and inflammation in OGD/R-treated PC12 cells.

Apoptosis is considered as one of the most important cellular processes in OGD/R-induced injury. Therefore, the apoptosis of OGD/R-treated PC12 cells was determined. As shown in Fig. 3A and B, cell apoptosis was enhanced among OGD/R-treated PC12 cells; however, PF alleviated cell apoptosis in a dose-dependent manner. Furthermore, the expression of the pro-apoptotic proteins Bax, cleaved caspase-9 and cleaved PARP was notably upregulated, while that of the anti-apoptotic protein $\mathrm{Bcl}-2$ was downregulated by OGD/R. These effects were reversed by increasing concentrations of PF (Fig. 3C). Collectively, these results revealed that $\mathrm{PF}$ attenuated oxidative stress, inflammation and apoptosis in OGD/R-treated PC12 cells.

PF activates JAK2/STAT3 signaling in OGD/R-treated PC12 cells. Subsequently, the possible association between PF and the JAK2/STAT3 signaling pathway was evaluated by western blot analysis. It was found that the protein expression levels 

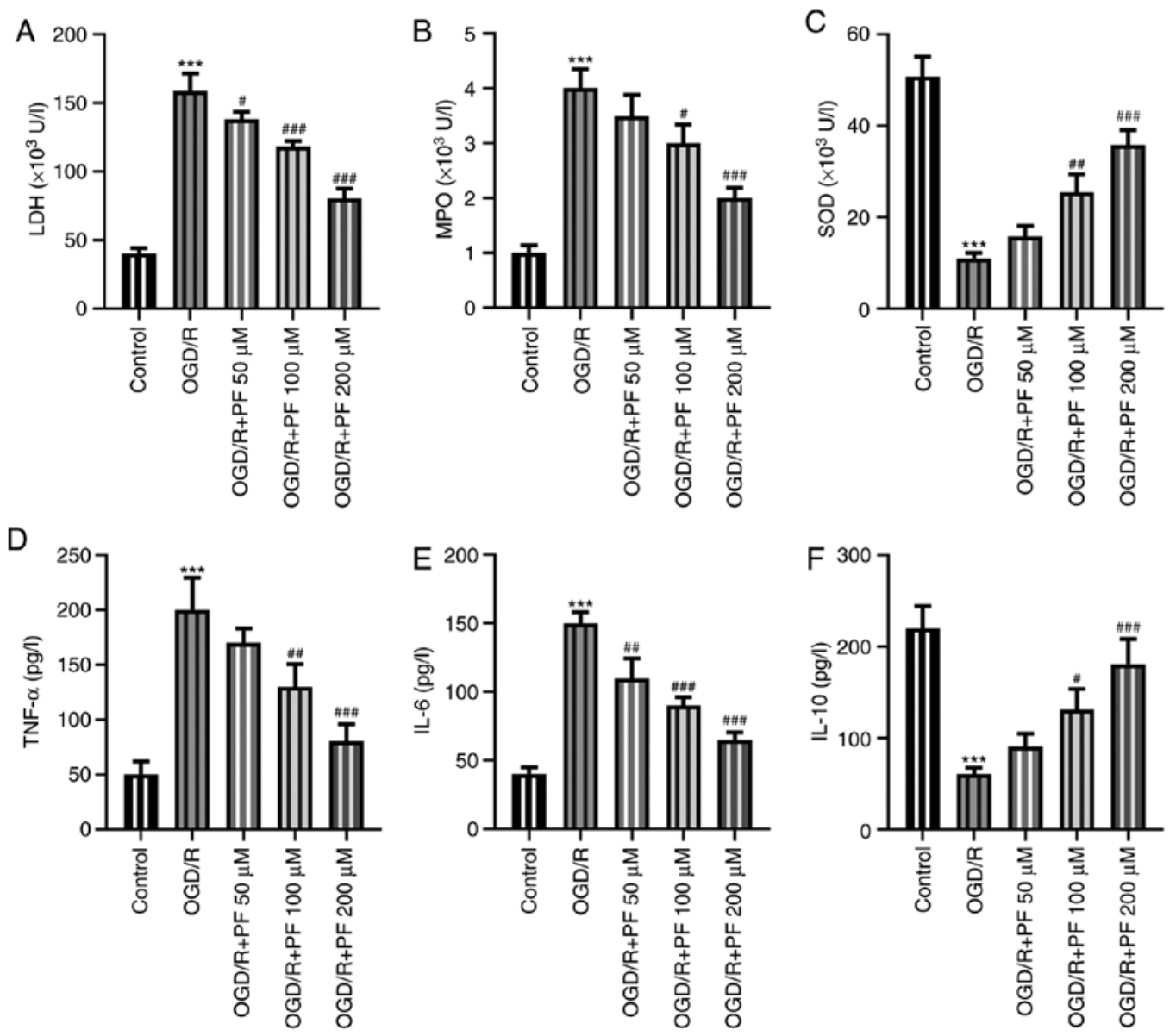

Figure 2. PF alleviates oxidative stress and inflammation in OGD/R-treated PC12 cells. Activity of (A) LDH, (B) MPO and (C) SOD in PC12 cells subjected to OGD/R and treated with PF was measured using commercial kits. ELISA was used to determine the secretion levels of the inflammatory factors (D) TNF- $\alpha$ (E) IL-6 and (F) IL-10. ${ }^{* * *} \mathrm{P}<0.001$ vs. the control group; ${ }^{*} \mathrm{P}<0.05,{ }^{\# \#} \mathrm{P}<0.01$, ${ }^{\# \# \#} \mathrm{P}<0.001$ vs. the $\mathrm{OGD} / \mathrm{R}$ group. $\mathrm{PF}$, paeoniflorin; OGD/R, oxygen-glucose deprivation/reoxygenation; LDH, lactate dehydrogenase; MPO myeloperoxidase; SOD, superoxide dismutase.

of phosphorylated (p)-JAK2 and p-STAT3 were significantly decreased in the OGD/R-treated group compared with the untreated group (Fig. 4). In addition, treatment with increasing doses of PF gradually enhanced the expression levels of p-JAK2 and p-STAT3 compared with the OGD/R group. These results suggested that the JAK2/STAT3 signaling pathway may be activated by PF. To better evaluate the effects of $\mathrm{PF}$, the concentration of $200 \mu \mathrm{M}$ was selected for the subsequent experiments.

FLLL32 abrogates the inhibitory effects of PF on oxidative stress, inflammation and apoptosis in $O G D / R$-treated PC12 cells. Subsequently, PC12 cells subjected to OGD/R were co-treated with the JAK2/STAT3 signaling inhibitor, FLLL32, after PF addition, to verify whether PF exerted its protective effects on these cells via the JAK2/STAT3 signaling pathway. As shown in Fig. 5A, the OGD/R-mediated reduced PC12 cell viability was restored by PF, while co-treatment with FLLL32 decreased the cell viability. The effect of PF on alleviating oxidative stress and inflammatory responses was partially counteracted by FLLL32 intervention (Fig. 5B-G). As regards cell apoptosis, $\mathrm{PF}$ suppressed the OGD/R-mediated PC12 cell apoptosis, which was further promoted by FLLL32 (Fig. 6A and B). Consistent with the previous findings, treatment with FLLL32 markedly reduced the expression levels of Bcl-2, which was accompanied by the upregulated expression of Bax, cleaved caspase- 9 and cleaved PARP, compared with the OGD/R + PF group. The aforementioned findings verified that FLLL32 could abrogate the protective effects of PF on OGD/R-treated PC12 cells.

\section{Discussion}

In vivo and in vitro studies have suggested that $\mathrm{PF}$ has potent anti-inflammatory and immunosuppressive properties, and it can reduce pain, joint swelling, synovial hypertrophy, bone erosion and cartilage degradation in arthritic rats (16-18). In addition, PF was shown to regulate the immune responses and increase the survival rate of septic rats (19). Furthermore, PF exerted antioxidant and anti-apoptotic effects in the treatment of cholestatic liver injury (20). Therefore, the present study sought to determine whether PF could also attenuate inflammation, oxidative stress and apoptosis in the treatment of ischemic stroke.

Oxidative stress is widely involved in several pathophysiological processes, such as aging, inflammation and tumorigenesis (21). A study suggested that pre-treatment of PC12 cells with PF may enhance cell viability and decrease the release of LDH (4). This finding was consistent with the results of the present study. MPO is a critical inflammatory 

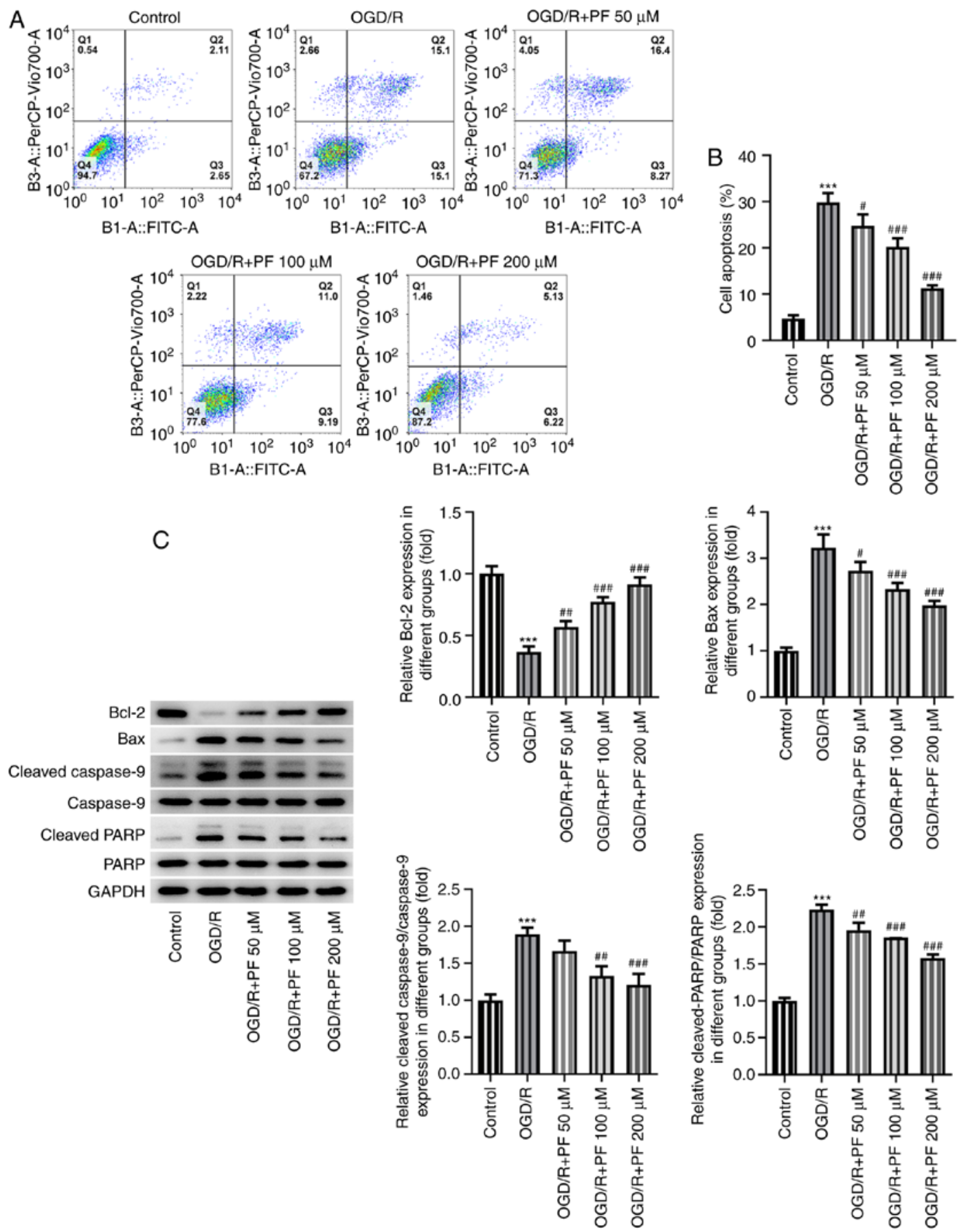

Figure 3. PF attenuates the apoptosis of OGD/R-treated PC12 cells. (A and B) Flow cytometry was used to evaluate the apoptosis of PC12 cells subjected to $\mathrm{OGD} / \mathrm{R}$ and treated with PF. (C) Western blot analysis was used to determine the expression levels of apoptosis-related proteins in OGD/R-treated PC12 cells ${ }^{* * * *} \mathrm{P}<0.001$ vs. the control group; ${ }^{\#} \mathrm{P}<0.05,{ }^{\# \#} \mathrm{P}<0.01,{ }^{* \# \#} \mathrm{P}<0.001$ vs. the $\mathrm{OGD} / \mathrm{R}$ group. $\mathrm{PF}$, paeoniflorin; $\mathrm{OGD} / \mathrm{R}$, oxygen-glucose deprivation/reoxygenation.

enzyme and therapeutic target triggering both oxidative stress and neuroinflammation during the pathological process of cerebral I/R injury (22). MPO is often upregulated in various inflammatory cells, while its inhibition has been associated with the development of a relatively protective environment from brain damage in a murine model of stroke (23). Furthermore, SOD can directly affect the antioxidant capacity $(24,25)$. Increased SOD activity was shown to enhance the protective mechanism against cerebral I/R injury in diabetic rats (26). Emerging evidence has suggested that $\mathrm{PF}$ can protect $\mathrm{HT}-22$ cells from $\mathrm{H}_{2} \mathrm{O}_{2}$-induced oxidative injury via regulating the expression of miR-135a (5). The present study demonstrated that PF alleviated oxidative stress in OGD/R-treated PC12 cells.

Previous studies have reported that PF exerts anti-inflammatory effects in order to regulate cellular functions (27-30). In the present study, treatment with PF also reduced the production of inflammatory cytokines. Apoptosis is one of the most important pathophysiological effects of ischemic stroke, and the aberrant expression of apoptosis-related proteins has been considered to serve as indicator or marker of cell death $(11,31)$. Other studies also supported the neuroprotective effects of PF, mediated by decreased Bax and increased Bcl-2 expression (4). Consistent with previous findings, in the present 

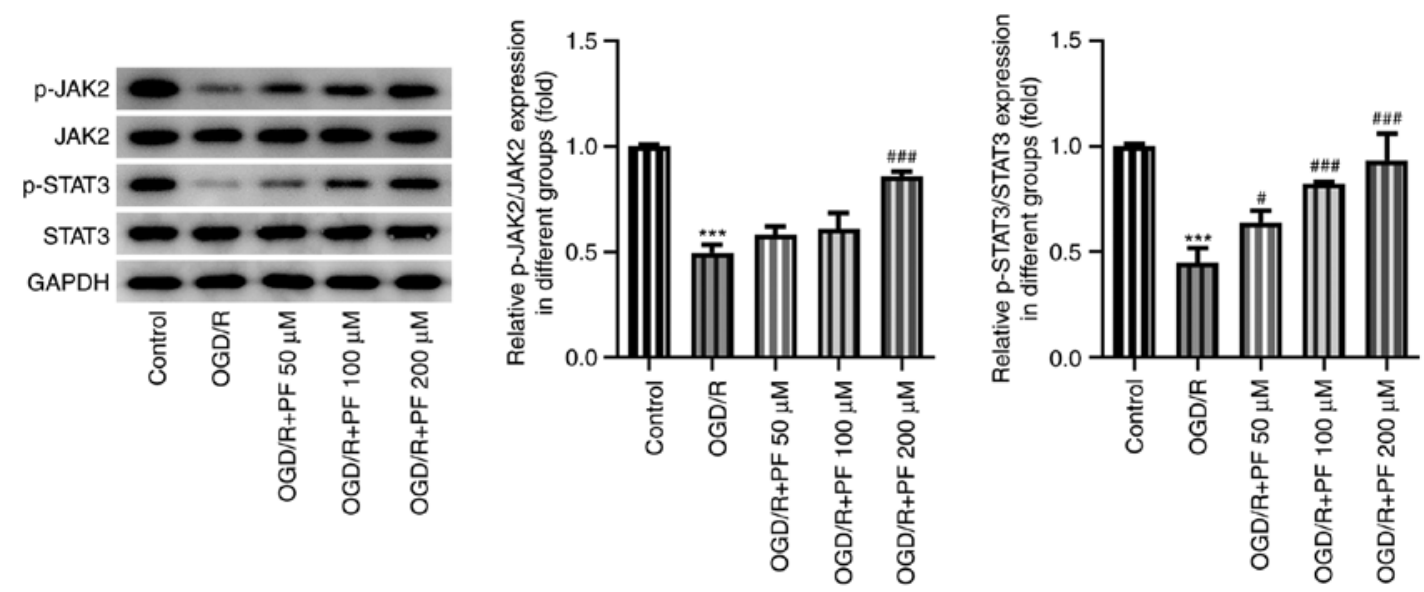

Figure 4. PF activates the JAK2/STAT3 signaling pathway in OGD/R-treated PC12 cells. The expression levels of the JAK2/STAT3 signaling-related proteins were detected by western blotting. ${ }^{* * *} \mathrm{P}<0.001$ vs. the control group; ${ }^{\#} \mathrm{P}<0.05,{ }^{\# \# \#} \mathrm{P}<0.001$ vs. the $\mathrm{OGD} / \mathrm{R}$ group. $\mathrm{PF}$, paeoniflorin; OGD/R, oxygen-glucose deprivation/reoxygenation; JAK2, Janus kinase 2; STAT3, signal transducer and activator of transcription 3.
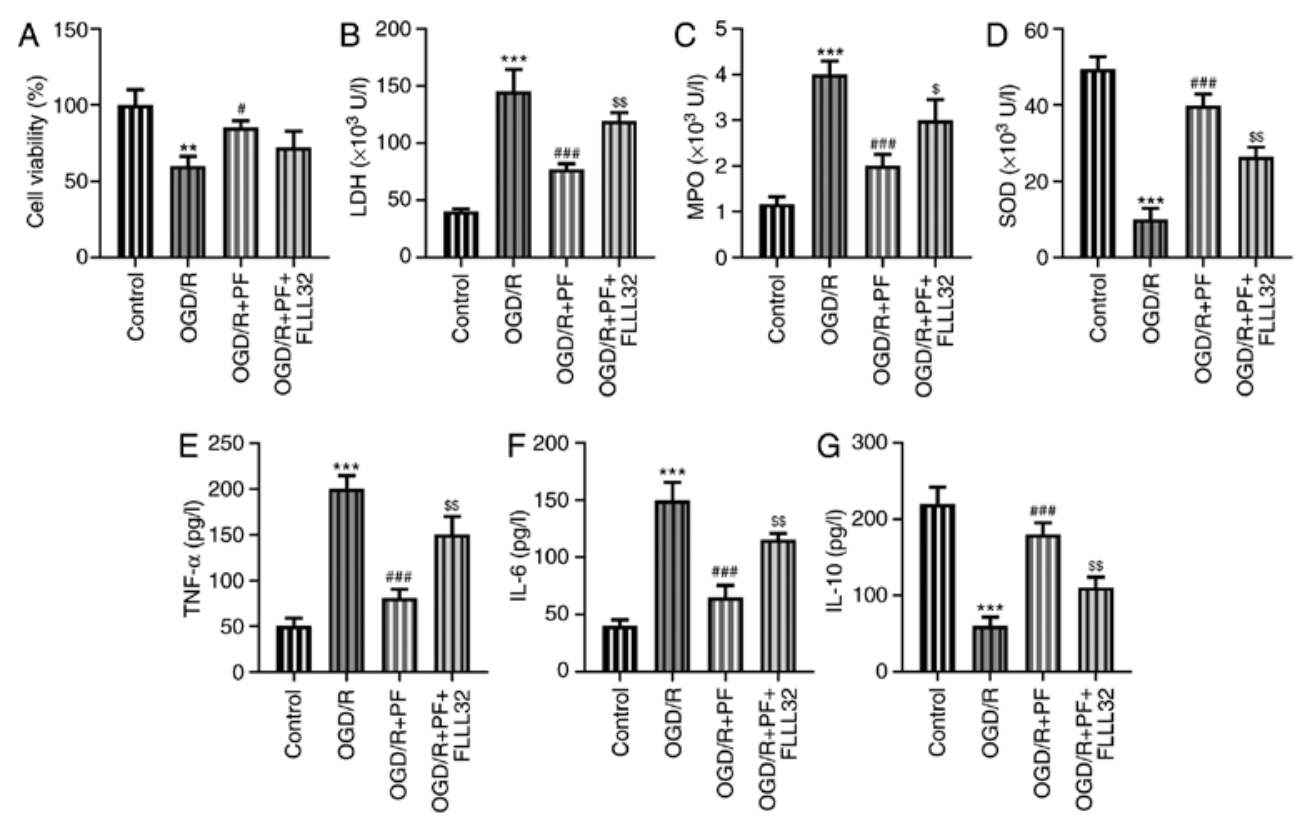

Figure 5. FLLL32, a specific inhibitor of Janus kinase 2/signal transducer and activator of transcription 3 signaling, abrogates the antioxidant and anti-inflammatory effects of PF on OGD/R-treated PC12 cells. (A) Cell viability was assessed using Cell Counting Kit-8 assay. The expression levels of the oxidative stress indicators (B) LDH, (C) MPO and (D) SOD were measured using commercial kits. The secretion levels of the inflammatory factors (E) TNF- $\alpha$ (F) IL-6 and (G) IL-10 in PC12 cells subjected to OGD/R and treated with PF and FLLL32 were determined using ELISA. ${ }^{* *} \mathrm{P}<0.01{ }^{* * * *} \mathrm{P}<0.001$ vs. the control group; ${ }^{\#} \mathrm{P}<0.05,{ }^{\# \# \#} \mathrm{P}<0.001$ vs. the OGD/R group; ${ }^{\$} \mathrm{P}<0.05,{ }^{\$ \$} \mathrm{P}<0.01$ vs. the $\mathrm{OGD} / \mathrm{R}+\mathrm{PF}$ group. $\mathrm{PF}$, paeoniflorin; OGD/R, oxygen-glucose deprivation/reoxygenation; $\mathrm{LDH}$, lactate dehydrogenase; MPO myeloperoxidase; SOD, superoxide dismutase.

study PF promoted the down- and upregulation of pro- and anti-apoptotic proteins, respectively, in OGD/R-treated PC12 cells. Therefore, $\mathrm{PF}$ attenuated oxidative stress, inflammation and apoptosis in OGD/R-treated PC12 cells.

It has been reported that the JAK2/STAT3 signaling pathway serves as a potent effector in attenuating cell death and apoptosis (32). JAK2/STAT3 signaling has also been associated with the occurrence and progression of several inflammatory diseases, including arthritis, apical periodontitis and Alzheimer's disease (33-35). JAK2 is essential for signaling through a variety of key class I cytokine receptors, such as IL-3, IL-6, interferon- $\gamma$ and leptin (36). The STAT transcription factors are crucial for cell fate. Among the members of the STAT family, STAT3 is involved in classic inflammatory diseases (37). A previous study demonstrated that the activation of the JAK2/STAT3 signaling pathway by different drugs protected mice from a series of pathological stress stimuli and I/R injury (38). In the present study, the expression levels of p-JAK2 and p-STAT3 were increased by $\mathrm{PF}$ in $\mathrm{OGD} / \mathrm{R}$-treated $\mathrm{PC} 12$ cells, suggesting that this signaling pathway may be activated by PF. Consistent with the findings of the present study, a previous study demonstrated that abolishment of the JAK2/STAT3 signaling pathway by a pharmacological inhibitor aggravated apoptosis and OGD/R injury (39).

Overall, the present study demonstrated that PF may protect PC12 cells from OGD/R-induced injury partly via activating JAK2/STAT3 signaling. Furthermore, the findings 

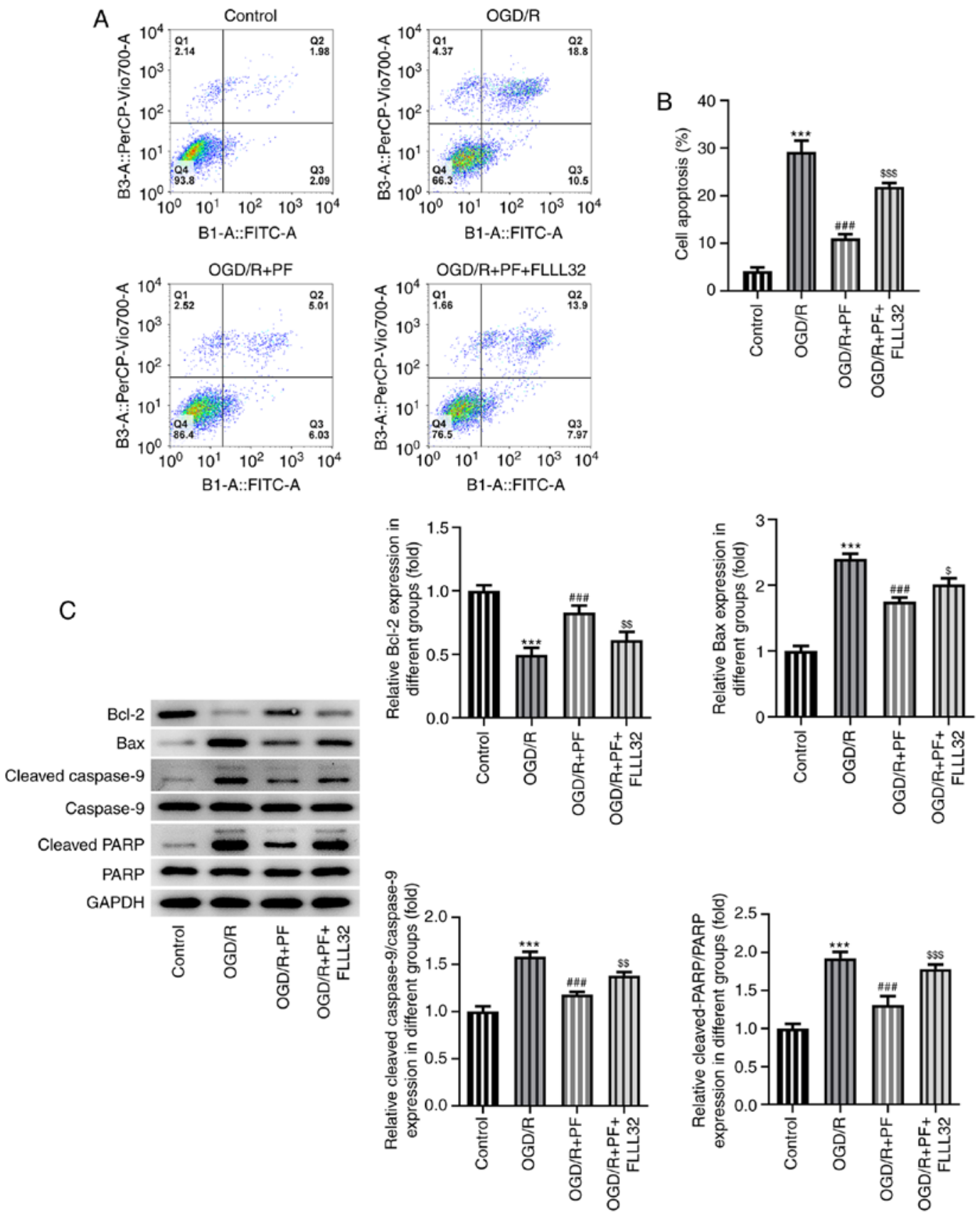

Figure 6. FLLL32, a specific inhibitor of Janus kinase 2/signal transducer and activator of transcription 3 signaling, attenuates the anti-apoptotic effects of PF on OGD/R-induced PC12 cells. (A and B) Flow cytometry was used to evaluate the apoptosis of PC12 cells subjected to OGD/R and co-treated with PF and FLLL32. (C) Western blot analysis was used to determine the expression levels of apoptosis-related proteins in PC12 cells subjected to OGD/R and treated with $\mathrm{PF}$ and FLLL32. ${ }^{* * *} \mathrm{P}<0.001$ vs. the control group; ${ }^{\# \# *} \mathrm{P}<0.001$ vs. the $\mathrm{OGD} / \mathrm{R}$ group; ${ }^{\$ \$} \mathrm{P}<0.01,{ }^{\$} \mathrm{P}<0.05,{ }^{\$ \$ \$} \mathrm{P}<0.001$ vs. the OGD/R + PF group. $\mathrm{PF}$, paeoniflorin; $\mathrm{OGD} / \mathrm{R}$, oxygen-glucose deprivation/reoxygenation; PARP, poly(adenosine diphosphate-ribose) polymerase.

of the present study may provide a better understating of the mechanism through which PF alleviates the adverse effects of ischemic stroke and indicate a promising approach to treating this disease. The lack of studies in vivo is a limitation of the present research. Based on the present findings, the effects of PF on the functional recovery of animals with cerebral I/R injury will be studied in a middle cerebral artery occlusion and reperfusion rat model. Additionally, whether PF can inhibit oxidative stress, inflammation and apoptosis and the regulatory effects of JAK2/STAT3 signaling and other potential pathways will be further investigated in the following experiments.

\section{Acknowledgements}

Not applicable.

\section{Funding}

No funding was received.

\section{Availability of materials and data}

The datasets used and/or analyzed during the current study are available from the corresponding author on reasonable request. 


\section{Authors' contributions}

$\mathrm{ZZ}$ and WY searched the literature, designed the experiments and performed the experiments. $\mathrm{ZZ}$ analyzed, interpreted the data and wrote the manuscript. WY revised the manuscript. $\mathrm{ZZ}$ and WY confirmed the authenticity of all the raw data. Both authors have read and approved the final manuscript.

\section{Ethics approval and consent to participate}

Not applicable.

\section{Patient consent for publication}

Not applicable.

\section{Competing interests}

The authors declare that they have no competing interests.

\section{References}

1. Martini ML, Neifert SN, Lara-Reyna JJ, Shuman WH, Ladner TR, Hardigan TH, Fifi JT, Mocco J and Yaeger KA Trials in thrombectomy for acute ischemic stroke: Describing the state of clinical research in the field. Clin Neurol Neurosurg 200 106360, 2021.

2. Chen H, Yoshioka H, Kim GS, Jung JE, Okami N, Sakata H, Maier CM, Narasimhan P, Goeders CE and Chan PH: Oxidative stress in ischemic brain damage: Mechanisms of cell death and potential molecular targets for neuroprotection. Antioxid Redox Signal 14: 1505-1517, 2011.

3. Bavarsad K, Barreto GE, Hadjzadeh MA and Sahebkar A: Protective effects of curcumin against ischemia-reperfusion injury in the nervous system. Mol Neurobiol 56: 1391-1404, 2019.

4. Chen A, Wang H, Zhang Y, Wang X, Yu L, Xu W, Xu W and Lin Y: Paeoniflorin exerts neuroprotective effects against glutamate induced PC12 cellular cytotoxicity by inhibiting apoptosis. Int J Mol Med 40: 825-833, 2017.

5. Zhai A, Zhang Z and Kong X: Paeoniflorin alleviates $\mathrm{H}_{2} \mathrm{O}_{2}$-induced oxidative injury through down-regulation of microRNA-135a in HT-22 cells. Neurochem Res 44: 2821-2831, 2019.

6. Wen Z, Hou W, Wu W, Zhao Y, Dong X, Bai X, Peng L and Song L: 6'-O-Galloylpaeoniflorin attenuates cerebral ischemia reperfusion-induced neuroinflammation and oxidative stress via PI3K/Akt/Nrf2 activation. Oxid Med Cell Longev 2018: 8678267, 2018.

7. Liu H, Yu C, Xu T, Zhang X and Dong M: Synergistic protective effect of paeoniflorin and $\beta$-ecdysterone against rotenone-induced neurotoxicity in PC12 cells. Apoptosis 21: 1354-1365, 2016.

8. Shio MT, Christian JG, Jung JY, Chang KP and Olivier M: $\mathrm{PKC} / \mathrm{ROS}$-mediated NLRP3 inflammasome activation is attenuated by leishmania zinc-metalloprotease during Infection. PLoS Negl Trop Dis 9: e0003868, 2015.

9. Li J, Ji X, Zhang J, Shi G, Zhu X and Wang K: Paeoniflorin attenuates $\mathrm{A} \beta 25$-35-induced neurotoxicity in $\mathrm{PC} 12$ cells by preventing mitochondrial dysfunction. Folia Neuropathol 52: 285-290, 2014

10. Wang D, Wong HK, Feng YB and Zhang ZJ: Paeoniflorin, a natural neuroprotective agent, modulates multiple anti-apoptotic and pro-apoptotic pathways in differentiated PC12 cells. Cell Mol Neurobiol 33: 521-529, 2013.

11. Hou Y, Wang K, Wan W, Cheng Y, Pu X and Ye X: Resveratrol provides neuroprotection by regulating the JAK2/STAT3/PI3K/ AKT/mTOR pathway after stroke in rats. Genes Dis 5: 245-255, 2018.

12. de Couto G, Liu W, Tseliou E, Sun B, Makkar N, Kanazawa H, Arditi M and Marbán E: Macrophages mediate cardioprotective cellular postconditioning in acute myocardial infarction. J Clin Invest 125: 3147-3162, 2015.
13. Li X, Wang Y, Wang K and Wu Y: Renal protective effect of Paeoniflorin by inhibition of JAK2/STAT3 signaling pathway in diabetic mice. Biosci Trends 12: 168-176, 2018.

14. Yang B, Zang LE, Cui JW, Zhang MY, Ma X and Wei LL: Melatonin plays a protective role by regulating miR-26a-5p-NRSF and JAK2-STAT3 pathway to improve autophagy, inflammation and oxidative stress of cerebral ischemia-reperfusion injury. Drug Des Devel Ther 14: 3177-3188, 2020.

15. Yuan J, Zeng L, Sun Y, Wang N, Sun Q, Cheng Z and Wang Y: $\mathrm{SH} 2 \mathrm{~B} 1$ protects against OGD/R induced apoptosis in $\mathrm{PC} 12$ cells via activation of the JAK2/STAT3 signaling pathway. Mol Med Rep 18: 2613-2620, 2018.

16. Zhang LL, Wei W, Wang NP, Wang QT, Chen JY, Chen Y, $\mathrm{Wu} \mathrm{H}$ and $\mathrm{Hu} \mathrm{XY}$ : Paeoniflorin suppresses inflammatory mediator production and regulates $G$ protein-coupled signaling in fibroblast-like synoviocytes of collagen induced arthritic rats. Inflamm Res 57: 388-395, 2008.

17. Ling L, Tong J and Zeng L: Paeoniflorin improves acute lung injury in sepsis by activating Nrf2/Keap1 signaling pathway. Sichuan Da Xue Xue Bao Yi Xue Ban 51: 664-669, 2020 (In Chinese).

18. Zhou H, Bian D, Jiao X, Wei Z, Zhang H, Xia Y, He Y and Dai Y: Paeoniflorin protects against lipopolysaccharide-induced acute lung injury in mice by alleviating inflammatory cell infiltration and microvascular permeability. Inflamm Res 60: 981-990, 2011.

19. Jiang WL, Chen XG, Zhu HB, Gao YB, Tian JW and Fu FH Paeoniflorin inhibits systemic inflammation and improves survival in experimental sepsis. Basic Clin Pharmacol Toxicol 105: 64-71, 2009.

20. Wei S, Ma X, Niu M, Wang R, Yang T, Wang D, Wen J, Li H and Zhao Y: Mechanism of paeoniflorin in the treatment of bile duct ligation-induced cholestatic liver injury using integrated metabolomics and network pharmacology. Front Pharmacol 11: 586806, 2020.

21. Poprac P, Jomova K, Simunkova M, Kollar V, Rhodes CJ and Valko M: Targeting free radicals in oxidative stress-related human diseases. Trends Pharmacol Sci 38: 592-607, 2017.

22. Chen S, Chen H, Du Q and Shen J: Targeting myeloperoxidase (MPO) mediated oxidative stress and inflammation for reducing brain ischemia injury: Potential application of natural compounds. Front Physiol 11: 433, 2020.

23. Yu G, Liang Y, Huang Z, Jones DW, Pritchard KA Jr and Zhang H: Erratum to: Inhibition of myeloperoxidase oxidant production by $\mathrm{N}$-acetyl lysyltyrosylcysteine amide reduces brain damage in a murine model of stroke. J Neuroinflammation 13: 166, 2016.

24. Briyal S, Gulati K and Gulati A: Repeated administration of exendin-4 reduces focal cerebral ischemia-induced infarction in rats. Brain Res 1427: 23-34, 2012.

25. Zhao L, Xu J, Wang Q, Qian Z, Feng W, Yin X and Fang Y: Protective effect of rhGLP-1 (7-36) on brain ischemia/reperfusion damage in diabetic rats. Brain Res 1602: 153-159, 2015.

26. Fang Y, Liu X, Zhao L, Wei Z, Jiang D, Shao H, Zang Y, Xu J, Wang Q, Liu Y, et al: RhGLP-1 (7-36) protects diabetic rats against cerebral ischemia-reperfusion injury via up-regulating expression of Nrf2/HO-1 and increasing the activities of SOD. Korean J Physiol Pharmacol 21: 475-485, 2017.

27. Dai X, Wang LW, Jia XY, Chang Y, Wu HX, Wang C and Wei W: Paeoniflorin regulates the function of human peripheral blood mononuclear cells stimulated by rhIL-1 $\beta$ by up-regulating Treg expression. Immunopharmacol Immunotoxicol 37: 252-257, 2015.

28. Jin L, Zhang LM, Xie KQ, Ye Y and Feng L: Paeoniflorin suppresses the expression of intercellular adhesion molecule-1 (ICAM-1) in endotoxin-treated human monocytic cells. Br J Pharmacol 164: 694-703, 2011.

29. Zhai T, Sun Y, Li H, Zhang J, Huo R, Li H, Shen B and Li N: Unique immunomodulatory effect of paeoniflorin on type I and II macrophages activities. J Pharmacol Sci 130: 143-150, 2016.

30. Shi D, Wang Q, Zheng H, Li D, Shen Y, Fu H, Li T, Mei H, Lu G, Qiu Y, et al: Paeoniflorin suppresses IL-6/Stat3 pathway via upregulation of Socs3 in dendritic cells in response to 1-chloro-2,4-dinitrobenze. Int Immunopharmacol 38: 45-53, 2016

31. Adams JM and Cory S: The Bcl-2 protein family: Arbiters of cell survival. Science 281: 1322-1326, 1998.

32. Shyu WC, Lin SZ, Chiang MF, Chen DC, Su CY, Wang HJ, Liu RS, Tsai $\mathrm{CH}$ and $\mathrm{Li} \mathrm{H}$ : Secretoneurin promotes neuroprotection and neuronal plasticity via the Jak $2 /$ Stat 3 pathway in murine models of stroke. J Clin Invest 118: 133-148, 2008.

33. Hossain E, Li Y and Anand-Srivastava MB: Role of JAK2/STAT3 pathway in angiotensin II-induced enhanced expression of Gialpha proteins and hyperproliferation of aortic vascular smooth muscle cells. Can J Physiol Pharmacol 99: 237-246, 2021. 
34. El-Ghafar OAMA, Helal GK and Abo-Youssef AM: Apixaban exhibits anti-arthritic effects by inhibiting activated factor $\mathrm{X}$-mediated JAK2/STAT3 and MAPK phosphorylation pathways. Inflammopharmacology 28: 1253-1267, 2020.

35. Pan S, Chen Y, Zhang X and Xie Y: The JAK2/STAT3 pathway is involved in dexmedetomidine-induced myocardial protection in rats undergoing cardiopulmonary bypass. Ann Transl Med 8: 483, 2020.

36. Wu QY, Ma MM, Fu L, Zhu YY, Liu Y, Cao J, Zhou P, Li ZY, Zeng LY, Li F, et al: Roles of germline JAK2 activation mutation JAK $2 \mathrm{~V} 625 \mathrm{~F}$ in the pathology of myeloproliferative neoplasms. Int J Biol Macromol 116: 1064-1073, 2018.

37. Leong PL, Andrews GA, Johnson DE, Dyer KF, Xi S, Mai JC, Robbins PD, Gadiparthi S, Burke NA, Watkins SF, et al: Targeted inhibition of Stat3 with a decoy oligonucleotide abrogates head and neck cancer cell growth. Proc Natl Acad Sci USA 100: 4138-4143, 2003.
38. $\mathrm{Li} \mathrm{L}, \mathrm{Li} \mathrm{H}$ and $\mathrm{Li} \mathrm{M}$ : Curcumin protects against cerebral ischemia-reperfusion injury by activating JAK2/STAT3 signaling pathway in rats. Int J Clin Exp Med 8: 14985-14991, 2015.

39. Li L, Sun L, Qiu Y, Zhu W, Hu K and Mao J: Protective effect of stachydrine against cerebral ischemia-reperfusion injury by reducing inflammation and apoptosis through $\mathrm{P} 65$ and JAK2/STAT3 signaling pathway. Front Pharmacol 11: 64, 2020.

This work is licensed under a Creative Commons Attribution-NonCommercial-NoDerivatives 4.0 International (CC BY-NC-ND 4.0) License. 